This item was submitted to Loughborough's Research Repository by the author.

Items in Figshare are protected by copyright, with all rights reserved, unless otherwise indicated.

\title{
Giant relaxation oscillations in a very strongly hysteretic superconductive quantum interference device ring-tank circuit system
}

PLEASE CITE THE PUBLISHED VERSION

http://dx.doi.org/10.1063/1.1398594

PUBLISHER

(C) American Institute of Physics (AIP)

VERSION

VoR (Version of Record)

LICENCE

CC BY-NC-ND 4.0

\section{REPOSITORY RECORD}

Clark, T.D., R.J. Prance, R. Whiteman, H. Prance, Mark J. Everitt, A.R. Bulsara, and J.F. Ralph. 2019. "Giant Relaxation Oscillations in a Very Strongly Hysteretic Superconductive Quantum Interference Device Ring-tank Circuit System". figshare. https://hdl.handle.net/2134/12082. 
This item was submitted to Loughborough's Institutional Repository (https://dspace.lboro.ac.uk/) by the author and is made available under the following Creative Commons Licence conditions.

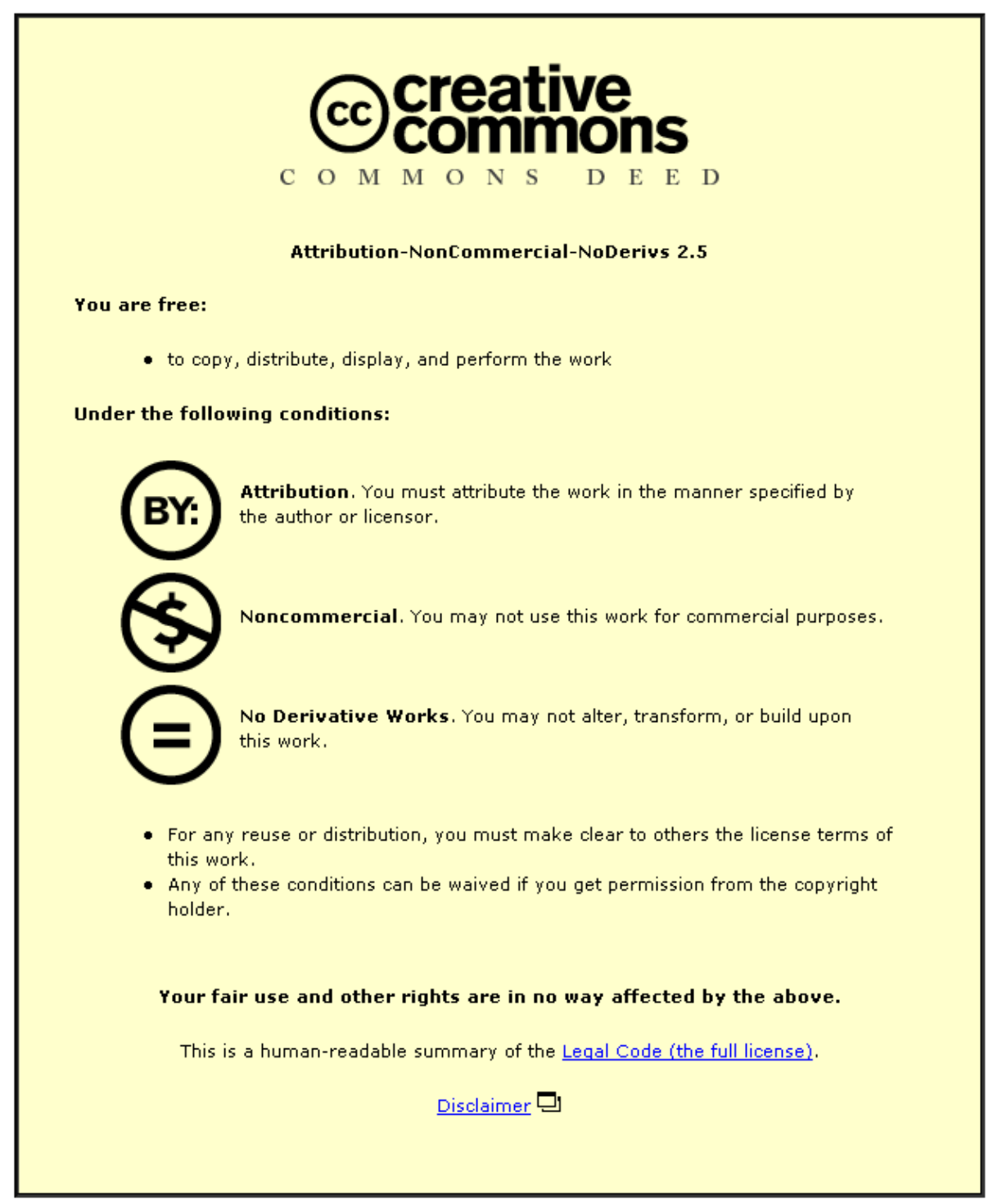

For the full text of this licence, please go to: http://creativecommons.org/licenses/by-nc-nd/2.5/ 


\section{AIP $\mid$ Appolied Physics}

Giant relaxation oscillations in a very strongly hysteretic superconductive quantum interference device ring-tank circuit system

T. D. Clark, R. J. Prance, R. Whiteman, H. Prance, M. J. Everitt et al.

Citation: J. Appl. Phys. 90, 3042 (2001); doi: 10.1063/1.1398594

View online: http://dx.doi.org/10.1063/1.1398594

View Table of Contents: http://jap.aip.org/resource/1/JAPIAU/v90/i6

Published by the American Institute of Physics.

Additional information on J. Appl. Phys.

Journal Homepage: http://jap.aip.org/

Journal Information: http://jap.aip.org/about/about_the_journal

Top downloads: http://jap.aip.org/features/most_downloaded

Information for Authors: http://jap.aip.org/authors 


\title{
Giant relaxation oscillations in a very strongly hysteretic superconductive quantum interference device ring-tank circuit system
}

\author{
T. D. Clark, ${ }^{\text {a) }}$ R. J. Prance, R. Whiteman, H. Prance, and M. J. Everitt \\ Physical Electronics Group, School of Engineering, University of Sussex, Brighton, Sussex BN1 9QT, \\ United Kingdom \\ A. R. Bulsara \\ Space and Naval Warfare Systems Centre, San Diego, California 92152-5001 \\ J. F. Ralph \\ Department of Electrical Engineering and Electronics, Liverpool University, Brownlow Hill, Liverpool, \\ L69 3GJ, United Kingdom
}

(Received 15 June 2001; accepted for publication 6 July 2001)

In this article, we show that the radio frequency (rf) dynamical characteristics of a very strongly hysteretic superconducting quantum interference device (SQUID) ring, coupled to a rf tank circuit resonator, display relaxation oscillations. We demonstrate that the overall form of these characteristics, together with the relaxation oscillations, can be modeled accurately by solving the quasiclassical nonlinear equations of motion for the system. We suggest that in these very strongly hysteretic regimes, SQUID ring-resonator systems may find application in logic and memory devices. (C) 2001 American Institute of Physics. [DOI: 10.1063/1.1398594]

\section{INTRODUCTION}

Over the years, the nonlinear, dynamical properties of superconducting quantum interference device (SQUID) rings (i.e., thick superconducting rings containing either one or two Josephson weak link devices) have formed the basis of a range of technologically important devices, ${ }^{1}$ most recently through the phenomenon of stochastic resonance. ${ }^{2}$ Apart from these applications, the study of the physics of the SQUID rings themselves ${ }^{1,3-5}$ has added considerably to the general field of nonlinear dynamics. As studied previously, these dynamical properties have been due to the quasiclassical behavior of SQUID rings in the regime where quantum processes can be neglected. However, of late, much interest has been shown in the use of SQUID rings as purely quantum devices in possible quantum technologies. ${ }^{6}$ This interest has been encouraged by recent experimental work on superposition states in weak link circuits ${ }^{7-13}$ and, in particular, on SQUID rings. ${ }^{14,15}$ Inevitably, at some stage, quantum circuits are required to interact with classical probe circuits if information about their quantum state/evolution is to be extracted. As we have shown, for the case of the SQUID ring, this interaction leads to the growth of nonlinear behavior in the classical part of the system. ${ }^{5,16-18}$ Thus, from this perspective, nonlinear dynamics always have a crucial role to play in the description, quantum or quasiclassical, of SQUID rings. In this article, we shall demonstrate that, even with the quite understandable attention being paid to SQUID rings at the quantum level, there is much still to be explored in the quasiclassical regime. This can add greatly to our knowledge of nonlinear systems and could lead to alternative and powerful applications.

${ }^{a)}$ Electronic mail: t.d.clark@ sussex.ac.uk
In the description of SQUID rings ${ }^{1}$ it is customary (from this quasiclassical viewpoint) to identify regimes of behavior in terms of the parameter $\beta\left(=2 \pi \Lambda I_{c} / \Phi_{0}\right)$, where $\Lambda_{s}$ is the ring inductance, $I_{c}$ is the (Josephson) critical current of the weak link in the ring, and $\Phi_{0}=h / 2 e$. In the presence of an external magnetic flux $\Phi_{x}$, the response of a SQUID ring is to set up a screening supercurrent $I_{s}\left(\Phi_{x}\right)$. The functional form of $I_{s}\left(\Phi_{x}\right)$ depends on the value of $\beta$. Thus, for $\beta$ $\leqslant 1, I_{s}$ is always single valued in $\Phi_{x}$ while for $\beta>1$, this current is multivalued and hysteretic in $\Phi_{x}$. In this latter regime, $I_{s}\left(\Phi_{x}\right)$ can be viewed as a set of approximately diamagnetic branches with switching between adjacent branches (in the absence of noise) occurring when $I_{s}$ reaches $\pm I_{c}$. Given this branch switching, hysteresis loops will be swept out, with concomitant dissipation, when $I_{s}$ exceeds $\pm I_{c}$. Devices which utilize the properties of single weak link SQUID rings, such as magnetometers, ${ }^{1}$ are usually operated at low $\beta$ values (e.g., 2 to 5). In operation as magnetometers, single weak link SQUID rings are typically coupled inductively to a radio frequency (rf) ( $\mathrm{rf} \approx 20 \mathrm{MHz}$ ), parallel inductor-capacitor, tank circuit which is excited using a rf current source. This arrangement is shown schematically in Fig. 1. As is well known, the rf voltage developed across the tank circuit is a function of both the rf magnetic flux amplitude and the static magnetic flux $\left(\Phi_{x \text { stat }}\right)$ applied to the ring. This static flux dependence is periodic in $\Phi_{0}$, hence the use of the SQUID ring-tank circuit system in magnetometery.

The dynamical behavior of a SQUID ring coupled to an external resonant circuit is governed, ultimately, by the form of its potential $U\left(\Phi, \Phi_{x}\right)$. Quasiclassically ${ }^{1}$ (i.e., when the effective mass of the ring - the weak link capacitance $C$-is large, typically $10^{-12}$ to $10^{-14} \mathrm{~F}$ for $4 \mathrm{~K}$ operation) this potential has the form 


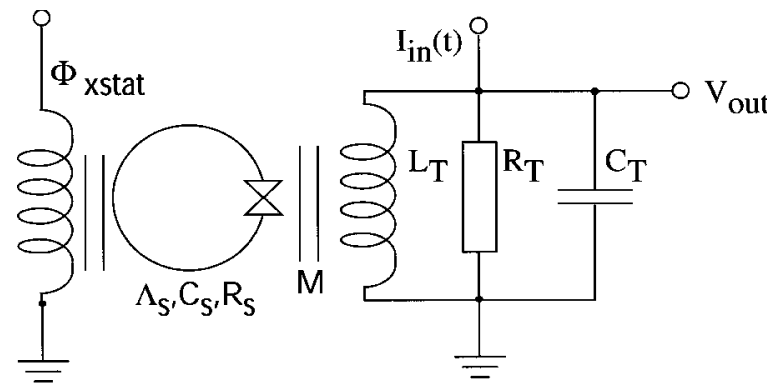

FIG. 1. Schematic of the coupled SQUID ring-rf tank circuit system also showing the static magnetic flux bias $\Phi_{x \text { stat }}$ applied to the ring.

$$
U\left(\Phi, \Phi_{x}\right)=\frac{\left(\Phi-\Phi_{x}\right)^{2}}{2 \Lambda_{s}}-\left(\frac{I_{c} \Phi_{0}}{2 \pi}\right) \cos \left(\frac{2 \pi \Phi}{\Phi_{0}}\right),
$$

where $\Phi\left(=\Phi_{x}+\Lambda I_{s}\right)$ is the total included flux in the ring. Clearly, if $\Phi_{x}$ changes slowly with time (i.e., slowly compared with any ring time constants), the ring will always remain at, or very close to, the minimum in $U\left(\Phi, \Phi_{x}\right)$. In dealing with ring-resonator dynamics, it has been customary to assume that this adiabatic constraint is satisfied, e.g., in the low $\beta$ rf SQUID magnetometer where the peak rf flux at the ring typically $\gtrsim \Phi_{0}(=\mu \varphi$ for a fraction $\mu$ of the tank circuit flux $\varphi$ coupled to the ring). The current $I_{s}$ set up in response to this flux then couples back to the tank circuit, and so on. This back reaction, which reflects $I_{s}\left(\Phi_{x}\right)$, affects the rf voltage $V_{\text {out }}$ of the tank circuit in a nonlinear way. With $I_{\text {in }}$ linearly amplitude modulated in time (e.g., at $\approx 100 \mathrm{~Hz}$ ), the result is the rf SQUID magnetometer $\left(V_{\text {out }}\right.$ verses $\left.I_{\text {in }}\right)$ characteristics. Following an initial linear riser in $V_{\text {out }}$ versus $I_{\text {in }}$, these characteristics consist of a set of voltage plateaux (steps) spaced at regular intervals along $I_{\text {in }}$, with period $\propto \Phi_{0} / \Lambda$. The $\Phi_{0}$-periodic modulation of these step features by a static (or quasistatic) applied flux $\Phi_{x \text { stat }}$ forms the basis of the rf SQUID magnetometer.

In this article, we report on the experimental and theoretical investigation of single weak link SQUID rings in the so-termed very strongly hysteretic regime, a previously little explored part of the parameter space of SQUID behavior. With the SQUID ring inductively coupled to a high quality factor $(Q) \operatorname{rf}(r f \approx 20 \mathrm{MHz})$ resonant circuit, we show that quite remarkable nonlinear phenomena (large amplitude relaxation oscillations) can develop. We demonstrate that these dynamics are the exact solutions of the coupled nonlinear equations of motion of the system.

\section{SQUID RING-TANK CIRCUIT DYNAMICS IN THE STRONGLY HYSTERETIC REGIME}

\section{A. Quasiclassical equations of motion}

The quasiclassical description of the ring-tank circuit system is based on the resistively shunted junction plus capacitance $[(\mathrm{RSJ})+C]$ model of a weak link. ${ }^{1,5}$ Here, the supercurrent channel through the link is in parallel with a normal current channel (resistance $R_{S}$ ) and a capacitance $C_{S}$. In the model, the normal channel turns on when the link current $I>I_{c}$. With representative values of $C_{s}$ from $10^{-12}$ to
$10^{-13} \mathrm{~F}$, generally accepted resistances $R_{S}$ are in the 10 to $100 \Omega$ range. ${ }^{19}$ With this in mind, the coupled ring-tank circuit equations of motion are ${ }^{1,20}$

$$
\begin{array}{r}
\text { SQUID ring: } C_{s} \frac{d^{2} \Phi}{d t^{2}}+\frac{1}{R_{s}} \frac{d \Phi}{d t}+I_{c} \sin \left(\frac{2 \pi \Phi}{\Phi_{0}}\right) \\
+\frac{\Phi}{\Lambda_{s}\left(1-K^{2}\right)}=\frac{\mu \varphi}{\Lambda_{s}\left(1-K^{2}\right)},
\end{array}
$$

Tank circuit: $\quad C_{T} \frac{d^{2} \varphi}{d t^{2}}+\frac{1}{R_{T}} \frac{d \varphi}{d t}+\frac{\varphi}{L_{T}\left(1-K^{2}\right)}$

$$
=I_{\mathrm{in}}(t)+\frac{\mu \Phi}{\Lambda_{s}\left(1-K^{2}\right)}
$$

for tank circuit capacitance, inductance, and parallel resonance resistance $C_{T}, L_{T}$, and $R_{T}$, respectively, coupling constant $K=\sqrt{M^{2} / L_{T} \Lambda}$ for mutual inductance $M$, and Josephson current $I=I_{c} \sin \left(2 \pi \Phi / \Phi_{0}\right)$. It is apparent in Eq. (2) that there are several possible ring time constants $\left(C_{s} R_{s}, \Lambda_{s} / R s, 1 / \sqrt{\Lambda_{s} C_{s}}\right)$. Clearly, if the adiabatic constraint holds, and there are no time constant problems, the derivative terms in Eq. (2) can be neglected and Eq. (2) reduces to

$$
I_{c} \sin \left(2 \pi \Phi / \Phi_{0}\right)+\Phi / \Lambda_{s}\left(1-K^{2}\right)=\mu \varphi / \Lambda_{s}\left(1-K^{2}\right) .
$$

In the past, this, together with Eq. (3), has been the starting point of most dynamical descriptions of ring-tank circuit systems, at least for low $\beta$ SQUID rings. We note that in this linearized, small $\beta$, description $I_{c}$ (i.e., $\beta$ ) can be found from the point of onset in $I_{\text {in }}$ of the periodic step features in $V_{\text {out }}$ versus $I_{\text {in }}$ (the breakpoint) beyond the initial linear riser. ${ }^{21}$

As we emphasized recently, ${ }^{20}$ the adiabatic constraint breaks down when the ring is strongly hysteretic and underdamped. $^{22}$ Qualitatively, at $4 \mathrm{~K}$, transition widths for branch switching in $I_{s}\left(\Phi_{x}\right)$ are typically $\approx \Phi_{0} / 100$. At 20 $\mathrm{MHz}$, and at the rf drive levels used experimentally for a $\beta$ $\approx 50-100$ ring $\left(\mu \varphi \gtrsim 100 \Phi_{0}\right)$, the available time to switch between branches $\approx 10^{-12} \mathrm{~s}$, comparable to the shortest ring time constant. In this range of $\beta$, multiple level structures develop in the experimental $V_{\text {out }}$ versus $I_{\text {in }}$ characteristics. $^{20}$ These structures (plateaux of almost constant $V_{\text {out }}$ along $I_{\text {in }}$ ) are on a scale in $I_{\text {in }}$ large compared with standard rf SQUID magnetometer steps. However, these still maintain a form of $\Phi_{0}$ periodicity in $\Phi_{x \text { stat }}$. We also demonstrated numerically that these multiple level structure characteristics formed a particular class of solutions of Eqs. (2) and (3). Given the range of nonlinear phenomena that have already been recognized in ring-tank circuit systems, it would be surprising if these multiple level structures constituted the only alternate class of solutions in the high $\beta$ regime. At an even higher $\beta$, a second type of nonlinear dynamics [also a solution of Eqs. (2) and (3)] develops-relaxation oscillations in rf voltage against current - on scales very large $(\approx \times 10)$ compared with conventional SQUID magnetometer characteristics.

\section{B. Experimental $V_{\text {out }}$ versus $I_{\text {in }}$ characteristics}

In our experiments, we made use of Zimmerman, twohole, niobium point contact SQUID rings. ${ }^{23}$ The point contact weak links in these rings were adjusted, in situ, at liquid 


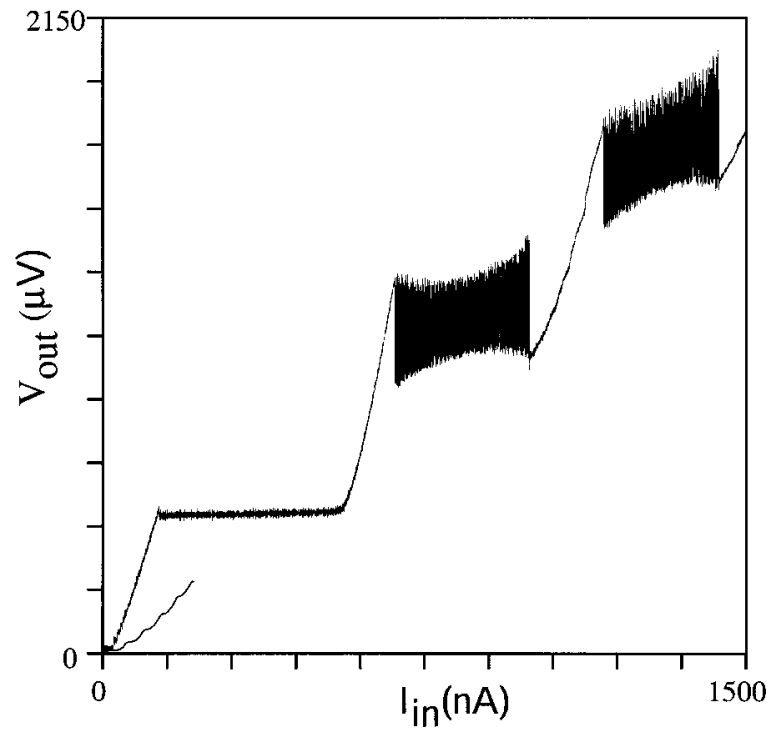

FIG. 2. Experimental $\left(4.2 \mathrm{~K} \quad \Phi_{x \mathrm{stat}}=n \Phi_{o}, \quad \Lambda=6 \times 10^{-10} \mathrm{H}, \quad L_{t}=6.3\right.$ $\times 10^{-8} \mathrm{H}, f_{\mathrm{rf}}=22.671 \mathrm{MHz}, K^{2}=0.008$ and $\left.Q=515\right) V_{\text {out }}$ versus $I_{\text {in }}$ characteristic for a niobium point contact SQUID ring-rf tank circuit system in the very highly hysteretic regime showing large scale relaxation oscillations are presented. Here, the triangular ramp amplitude modulation of the $\mathrm{rf}$ is at $18 \mathrm{~Hz}$ and the detection bandwidth of the receiver is $1 \mathrm{MHz}$.

helium temperatures, ${ }^{17,20}$ a technique which allows us to make weak links in a controlled way over a very wide range of $\beta$ values (e.g., $1<\beta<100$ ). In our experimental arrangement the tank circuit voltage $V_{\text {out }}$ (Fig. 1) was first amplified by a liquid helium cooled GaAs field effect transitor (FET) preamplifier (gain $\simeq 20 \mathrm{~dB}$, noise temperature $<10 \mathrm{~K}$ ). This signal was further boosted using a receiver of our own design and then diode detected. Overall, the receiver system (including the GaAs FET preamplifier) was designed to combine very low noise with very large dynamic range and slew rate, all three properties being required to observe the very strongly hysteretic behavior reported here.

In Fig. 2, we show a perfectly typical experimental diode detected $V_{\text {out }}$ versus $I_{\text {in }}$ SQUID magnetometer characteristic in this very strongly hysteretic regime. This was taken at 4.2 $\mathrm{K}$, in a bandwidth of $1 \mathrm{MHz}$, with $Q=515, K^{2}=0.008$, and a static bias flux $\Phi_{x \text { stat }}=n \Phi_{0}, n$ integer, supplied by a second coil coupled to the ring (Fig. 1). Here, adopting common practice, this characteristic was recorded at the $n \Phi_{o}$ bias frequency $f_{\mathrm{rf}}(22.671 \mathrm{MHz}$ in this case) equal to the resonant frequency of the ring-tank circuit system when $\Phi_{x s t a t}$ $=n \Phi_{0}$. As is standard for these dynamical (SQUID magnetometer) characteristics, the $\mathrm{rf}$ is amplitude modulated using a low frequency triangular ramp. In this example, the ramp frequency is $18 \mathrm{~Hz}$. As can be seen, the characteristic consists of a first feature, a flat step, followed by two further step features in which it is apparent that $V_{\text {out }}$ oscillates very rapidly as $I_{\text {in }}$ increases. Although not shown in Fig. 2, these oscillatory step structures, periodic in $I_{\text {in }}$, continued to repeat as $I_{\text {in }}$ was made larger. The rf voltage and current scales in Fig. 2 are made clear by the small, subsidiary characteristic also in Fig. 2. This was taken at $4.2 \mathrm{~K}$, with $\Phi_{x s t a t}$ $=n \Phi_{0}$, using the SQUID ring and tank circuit of the main characteristic but with the weak link in the ring adjusted to

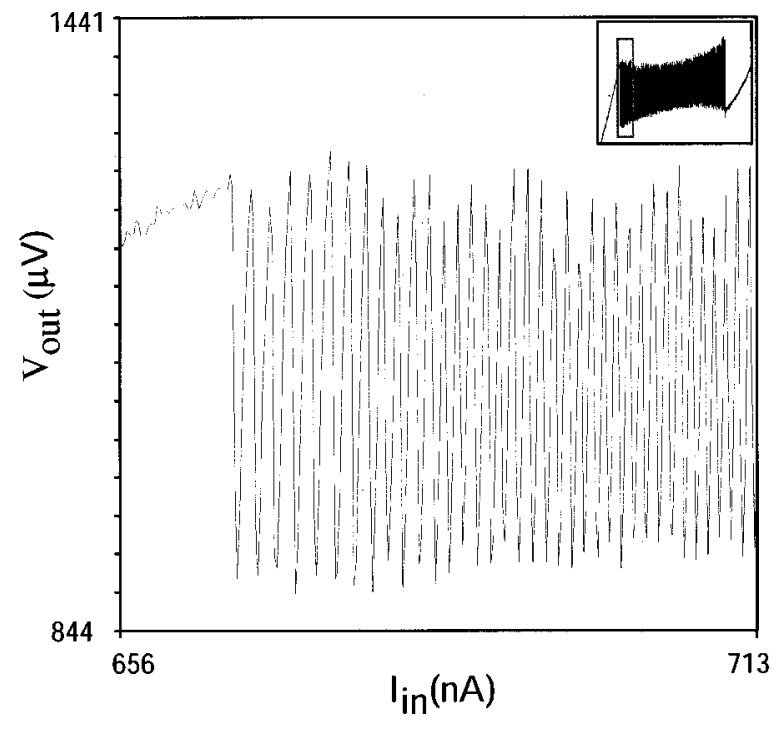

FIG. 3. Expanded region of the second plateau in Fig. 2 shows details of the relaxation oscillations.

give a $\beta$ just greater than unity, i.e., the regular rf SQUID magnetometer regime. It is obvious that the scalings of the main characteristic are very large indeed compared to those for a standard SQUID magnetometer. In Fig. 3, we show details of the second step structure of Fig. 2 along which the large scale oscillations first appear. Here, we have expanded the first section of this step structure (shown boxed in Fig. 3). In the expanded picture, it is apparent that $V_{\text {out }}$ is undergoing rapid oscillations as $I_{\text {in }}$ is increased. Furthermore, the spacing in $I_{\text {in }}$ between adjacent oscillations decreases monotonically as $I_{\text {in }}$ is swept across the step; this continues right across the step (not shown in Fig. 3). We note that the effect of a bias flux $\Phi_{x \text { stat }}$ on these oscillatory step structures was minimal, only changing the amplitude of the oscillations very weakly, with a periodicity of $\Phi_{0}$. Experimentally, a search of the first (flat) step in Fig. 1 did not reveal any voltage oscillations, only noise. Again, the effect of $\Phi_{x \text { stat }}$ was found to be negligible.

The wave form of Fig. 3 reflects a limit cycle consisting of a very slow build up (at least on the time scale of one $\mathrm{rf}$ oscillation $\left.2 \pi / \omega_{\text {rf }}\right)$ followed by an extremely fast discharge with energy drawn from the tank circuit. This is then followed by the next slow build up with energy supplied from the rf current source over many rf periods, and so on. These are, of course, relaxation oscillations which can be found in many physical situations where nonlinear dynamics are involved. ${ }^{24-27}$ For the particular case of the driven SQUID ring-tank circuit system of Fig. 3, the peak rf amplitude is increasing (linearly ramped with time) from the left-hand side to the right-hand side. Since the energy is made up from the rf current drive, this means that the time interval between successive oscillations shortens as $I_{\text {in }}$ increases, as is apparent in Fig. 3.

Without the combination of a very low noise receiver (including a liquid helium cooled GaAs FET preamplifier stage) and very large dynamic range/slew rate capability, the relaxation oscillations shown in Figs. 2 and 3 would not be observed. For example, reduced bandwidth or added noise, 
or a combination of both, can wash out these oscillations. What then remains are large (compared with ordinary SQUID magnetometer characteristics-see insert of Fig. 2), roughly constant rf voltage, current steps. This is presumably why these dynamics have not been observed in the past.

It is interesting to note that the relaxation oscillations observed by us in coupled SQUID ring-tank circuit systems in the very strongly hysteretic regime appear to have analogs in the phase slippage experiments which have been performed on superfluid helium systems. ${ }^{28-30}$ It seems clear that, putting the fundamental description of superfluid and superconducting condensates aside, the phase/flux slippage in the two systems is analogous. At a phenomenological level this means that the equations of motion governing the dynamics of the two ring-resonator systems are also analogous. In the highly hysteretic regime for phase slippage in superfluid rings containing weak link orifices, we would therefore expect to see (giant) relaxation oscillation phenomena and, perhaps, multilevel dynamics. ${ }^{20}$

\section{Numerical calculations}

The $V_{\text {out }}$ versus $I_{\text {in }}$ characteristics of Figs. 1 and 2 can be modeled using Eqs. (2) and (3) which can handle the problem of nonadiabaticity perfectly well. ${ }^{20}$ However, since for typical SQUID ring parameters $\left(C_{s} \approx 10^{-13} \mathrm{~F}, \quad \Lambda_{s}=6\right.$ $\times 10^{-10} \mathrm{H}, R_{s} \approx 10 \Omega$ ), the ring time constants will be 2 or more orders of magnitude shorter than the tank circuit time constant $(\cong 1 / 20 \mathrm{MHz})$, great care must be taken to ensure that accurate solutions are found. To solve Eqs. (2) and (3), we used fourth order Runge-Kutta with an adaptive step size algorithm. ${ }^{5,17,20}$ We were also careful to allow a sufficient number of steps per rf cycle to follow the changes in the SQUID ring accurately. For our comparison with the experiment, we set the rf drive frequency at the $\Phi_{x \text { stat }}=n \Phi_{0}$ resonant frequency (the pseudoresonant frequency). ${ }^{5,17,20}$ At this bias flux, the ring is at its most diamagnetic [with a ring magnetic susceptibility $\left.\chi\left(n \Phi_{0}\right)=\partial I_{s}\left(n \Phi_{0}\right) / \partial \Phi_{x} \cong-1\right]$ and $I_{s}$ is almost linear in $\Phi_{x}$. From the data of Fig. 2, we can make an estimate of $I_{c}$ (and hence $\beta$ ) from the start of the step features in the standard SQUID magnetometer compared with the (first) step feature in the large scale characteristics. ${ }^{20}$ This yields an $I_{c}$ for the large $\beta$ characteristic $\cong 80 \mu \mathrm{A}$ (i.e., a $\beta$ value in the 70 to 80 range). We have shown ${ }^{31}$ that knowing $I_{c}$ and the Fermi velocity of the material of the SQUID ring (here niobium), we can estimate $C$. For $I_{c} \simeq 100 \mu \mathrm{A}$, this gives $C_{s} \approx$ few $\times 10^{-13} \mathrm{~F}$. In addition, from singly connected current-voltage characteristics for point contact weak links, ${ }^{19}$ a typical value for $R_{s}$ at this $I_{c}$ is $\approx 10 \Omega$.

In Fig. 4, we show the results of solving Eqs. (2) and (3) to simulate the behavior of the ring-tank circuit system of Figs. 2 and 3, including a $4 \mathrm{~K}$ noise source. ${ }^{5,17,20}$ Given the very nonlinear nature of this coupled system, and the number of parameters involved, an exact match between theory and experiment has proved difficult. Nevertheless, we were able to reproduce the important features of the integer bias flux $V_{\text {out }}$ versus $I_{\text {in }}$ characteristic of Figs. 2 and 3 with $I_{c}$ $=80 \mu \mathrm{A}$ and best fit values $C_{s}=5 \times 10^{-12} \mathrm{~F}, R_{s}=10 \Omega$ and

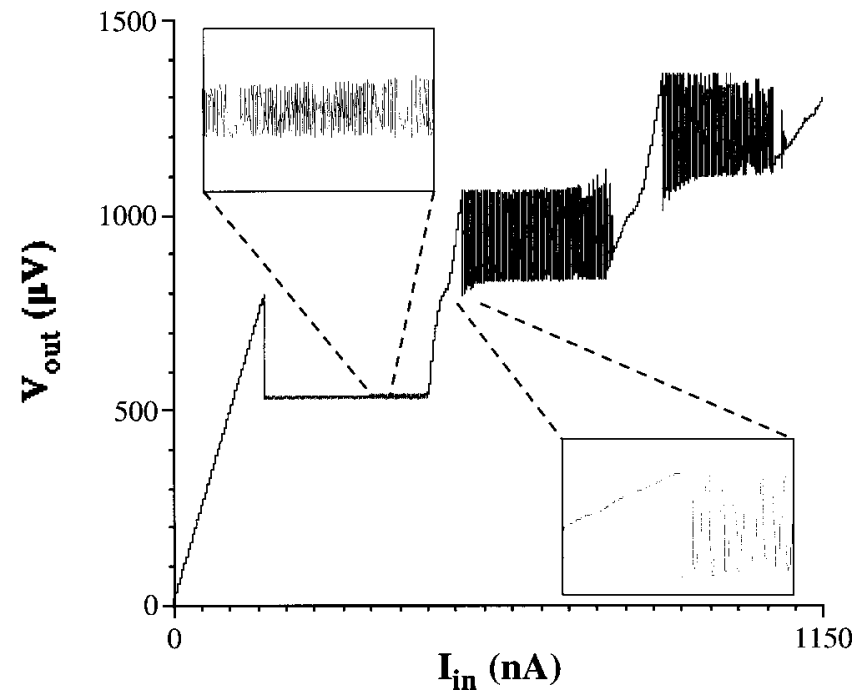

FIG. 4. Theoretical (RSJ $+C$ ) $V_{\text {out }}$ versus $I_{\text {in }}$ characteristic (with a $4 \mathrm{~K}$ tank circuit noise source added) to model the data of Figs. 2 and 3, setting $\Phi_{x \text { stat }}=n \Phi_{o}, \Lambda_{s}=6 \times 10^{-10} \mathrm{H}, L_{t}=6.3 \times 10^{-8} \mathrm{H}, K^{2}=0.008, Q=515$, and a best fit $f_{\mathrm{rf}}=25 \mathrm{MHz}$ is shown. Here, the effective triangular ramp amplitude modulation frequency is close to $30 \mathrm{~Hz}$.

$f_{\mathrm{rf}}=25 \mathrm{MHz}$. The other circuit parameters are, as for Figs. 2 and $3, Q=515, K^{2}=0.008, L_{T}=63 \mathrm{nH}$, while the effective triangular ramp frequency (converted from a rf current ramp in $\mu \mathrm{A} / \mathrm{s})$ is close to $30 \mathrm{~Hz}$. As can be seen, the computed solution contains both the first flat step and the relaxation oscillations on the second (and subsequent) step features of Fig. 2. Details of these (computed) oscillations are shown in the lower inset in Fig. 4. Also shown in the inset of Fig. 4, over a small range of $I_{\text {in }}$, is a highly expanded section of the first flat step. This too displays (weak) voltage oscillations which appear to be either aperiodic or multiperiodic. These oscillations were not seen experimentally, presumably because of limited signal to noise. If $I_{\text {in }}$ is fixed in the regions corresponding to the insets in Fig. 4-for the experimental data set of Fig. 2-and time series data are collected, these weak oscillations can be seen reflected in the (pseudo) phase portrait, as shown in Fig. 5(a). Here, the pseudophase portrait is defined as a plot of $V_{\text {out }}(t)$ against the time shifted $V_{\text {out }}(t+\tau)$ at all times $t$, where $\tau$ is a suitable time delay ( $10^{-4} \mathrm{~s}$ in this example). In the main part of Fig. 5(a), we show the pseudophase portrait, taken over a set of complete orbits, for the bias point in the upper inset. In the lower inset of Fig. 5, we display just one of these orbits. It is clear, for the first step, that this pseudophase portrait simply shows random fluctuations (noise) superimposed on a singly periodic orbit. This should be contrasted with the pseudophase portrait of Fig. 5(b) for $I_{\text {in }}$ biased onto the middle of the relaxation oscillations in the second $V_{\text {out }}$ versus $I_{\text {in }}$ step feature [shown in the upper inset of Fig. 5(b)]. Here, we have again plotted a set of orbits, with a single orbit of this set shown in the lower inset. As is apparent, both the set and the single orbit display the periodic behavior associated with the relaxation oscillations on the step.

What the theoretical computations of Fig. 4, the experimental data of Figs. 2 and 3, and the previously published multilevel structures,${ }^{20}$ demonstrate is that the form of the 

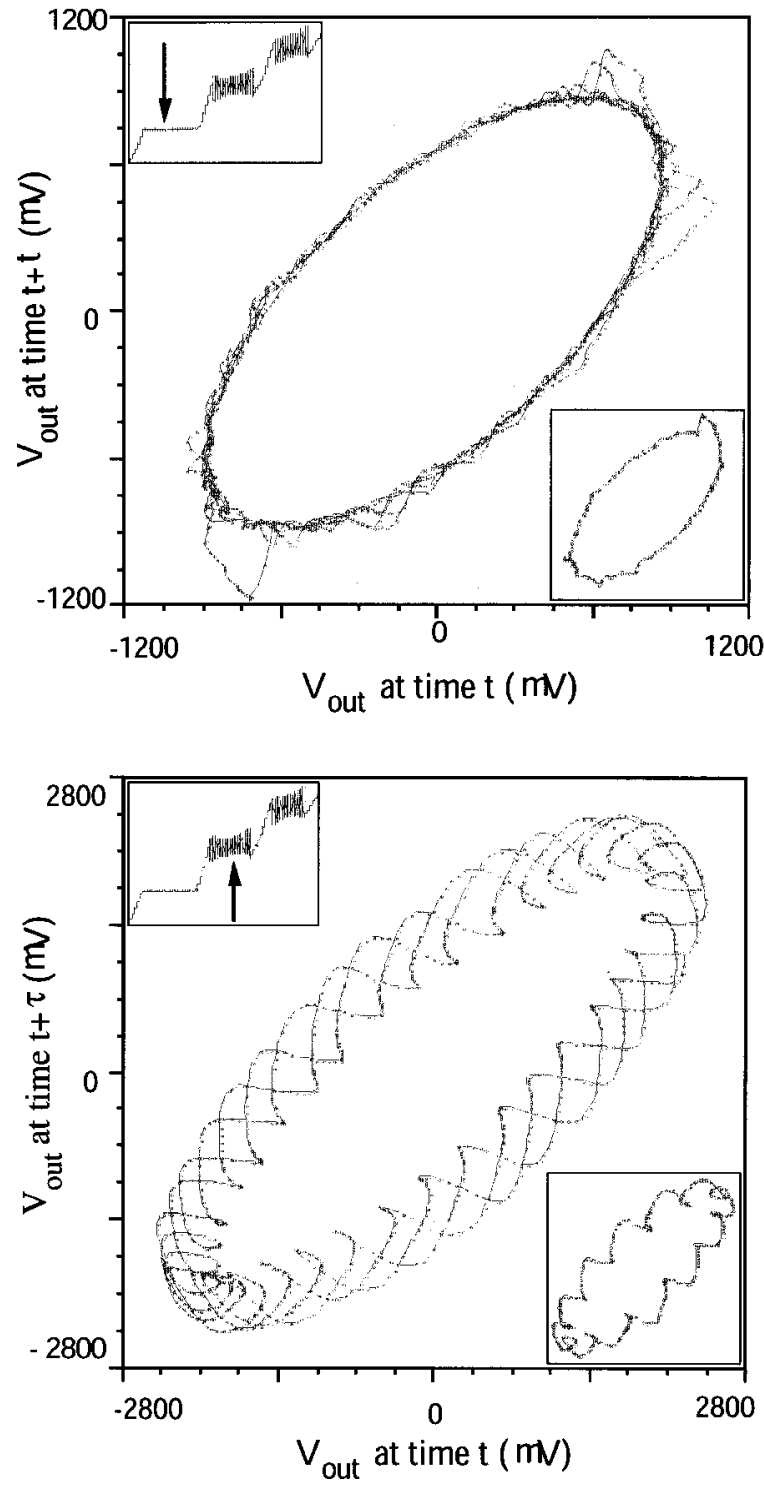

FIG. 5. Experimental pseudophase portraits $\left[V_{\text {out }}(t+\tau)\right.$, taken over sets of complete orbits, versus $V(t)]$ for the two $I_{\text {in }}$ bias points of Fig. 2 as shown arrowed in the insets are presented. Here, $\tau=10^{-4} \mathrm{~s}$ and $T=4.2 \mathrm{~K}$. The lower insets in (a) and (b) display single orbits of the data sets for the two bias points. The data was obtained in a detection bandwidth of $1 \mathrm{MHz}$ with an $18 \mathrm{~Hz}$ sweep frequency.

solutions to the coupled equations of motion (2) and (3) is critically dependent on parameter values. More specifically, it appears that in the very strongly hysteretic regime and above (i.e., $\beta \gtrsim 100$ ) the underdamping in the ring-tank circuit system becomes large enough to wash out multilevel structures completely, or almost completely. Physically, these structures arise (with stochastic jumping) from the system moving around $n$ hysteresis loops in $\Phi$ versus $\Phi_{x}$ (or $I_{s}$ versus $\left.\Phi_{x}\right)$ in $m$ rf cycles, $n$ and $m$ integer. ${ }^{22}$ When sufficiently underdamped, we assume that the system simply jumps between much larger values of $\Phi$ in $\Phi$ versus $\Phi_{x}$, followed by long recovery period over many rf cycles, i.e. a relaxation oscillation process.

\section{CONCLUSIONS}

The experimental data of Figs. 2 and 3 are typical of the $V_{\text {out }}$ versus $I_{\text {in }}$ characteristics found in the very strongly hys- teretic, and underdamped, regime $(\beta>100)$. These data can be modeled with considerable accuracy invoking the SQUID potential (1) and using the quasiclassical equations of motion (2) and (3), as can be seen from the computed $V_{\text {out }}$ versus $I_{\text {in }}$ characteristics of Fig. 4. Thus, from experiment and theory, we find that there exists another class of solution to the dynamics of the driven ring-tank circuit system in the very highly hysteretic regime, apart from multilevel structures. ${ }^{20}$ Since these (latter) structures clearly hold out the real possibility of quasiclassical multilevel logic and memory if these dynamics are well understood and can be controlled, there is every reason for opening up the phase space of behavior. Indeed, the potential for single flux quantum logic has already attracted a great deal of interest. ${ }^{32}$ It seems reasonable, therefore, that multilevel logic based on the manipulation of sets of discrete flux states of a SQUID ring in the strongly hysteretic regime will also generate interest. From the work reported here, it would appear that the change over between multilevel and relaxation solutions is quite subtle and sensitive to circuit parameters. From experiment and theory, we find that relaxation oscillation characteristics, very similar to those reported here, carry on to very much higher $\beta$ values $(>500)$. This, of course, points to these being a rather general class of solutions to Eqs. (2) and (3). Again, as with the earlier multilevel structures, ${ }^{20}$ these solutions do not arise in the linearized description of the system. We note, again from experiment and computation, that the multilevel structures ${ }^{20}$ found in the strongly hysteretic regime with $\beta \approx 50$ gradually fade away as $\beta$ increases, to be replaced by the relaxation oscillation characteristics reported in this article. In our opinion, even though other factors may be important in governing the already underdamped dynamics of this highly nonlinear system, this points to the damping becoming weaker as $\beta$ grows. It also seems clear that a rich new field of nonlinear dynamics exist which, as yet, has only been partially explored. From a historical perspective, it is also of interest that the original description of rf biased SQUID magnetometers ${ }^{21}$ was based on a relaxation oscillation model, albeit operating over very much shorter time intervals (a few rf periods), and on the rf voltage and current scales of conventional SQUID magnetometers. ${ }^{21}$ Nevertheless, relaxation oscillations have not been seen in the dynamics of these standard magnetometers.

\section{ACKNOWLEDGMENT}

The authors express thanks to the Engineering and Physical Sciences Research Council for its generous funding of this work.

${ }^{1}$ K. K. Likharev, Dynamics of Josephson Junctions and Circuits (Gordon and Breach, Sidney, 1986).

${ }^{2}$ See, for example, L. Gammaitoni, P. Hanggi, P. Jung, and F. Marchesoni, Rev. Mod. Phys. 70, 1 (1998); A. R. Bulsara, and L. Gammaitoni, Phys. Today 49, 39 (1996).

${ }^{3}$ A. R. Bulsara, J. Appl. Phys. 60, 2462 (1986).

${ }^{4}$ M. P. Soerensen, M. Barchelli, P. L. Christiansen, and A. R. Bishop, Phys. Lett. A 109, 347 (1985).

${ }^{5}$ T. D. Clark, J. F. Ralph, R. J. Prance, H. Prance, J. Diggins, and R. Whiteman, Phys. Rev. E 57, 4035 (1998).

${ }^{6} \mathrm{See}$, for example, Introduction to Quantum Computation and Information, 
edited by H. K. Lo, S. Popescu, and T. P. Spiller (World Scientific, Singapore, 1998).

${ }^{7}$ T. P. Orlando, J. E. Mooij, L. Tian, C. H. van der Wal, L. S. Levitov, S. Lloyd, and J. J. Mazo, Phys. Rev. B 60, 15398 (1999).

${ }^{8}$ Y. Makhlin, G. Schön, and A. Shnirman, Nature (London) 398, 305 (1999).

${ }^{9}$ D. Averin, Nature (London) 398, 748 (1999).

${ }^{10}$ R. Rouse, S. Han, and J. E. Lukens, Phys. Rev. Lett. 75, 1614 (1995).

${ }^{11}$ P. Silvestrini, B. B. Ruggierio, C. Granata, and E. Esposito, Phys. Lett. A 267, 45 (2000).

${ }^{12}$ Y. Nakamura, C. D. Chen, and J. S. Tsai, Phys. Rev. Lett. 79, 2328 (1997).

${ }^{13}$ Y. Nakamura, Y. A. Pashkin, and J. S. Tsai, Nature (London) 398, 786 (1999).

${ }^{14}$ C. H. van der Wal, A. C. J. ter Haar, F. K. Wilhem, R. N. Schouten, C. J. P. M. Harmans, T. P. Orlando, S. Lloyd, and J. E. Mooij, Science 290, 773 (2000).

${ }^{15}$ J. R. Friedman, V. Patel, W. Chen, S. K. Tolpygo, and J. E. Lukens, Nature (London) 406, 43 (2000).

${ }^{16}$ J. F. Ralph, T. P. Spiller, T. D. Clark, R. J. Prance, and H. Prance, Int. J. Mod. Phys. B 8, 2637 (1994).

${ }^{17}$ R. Whiteman, J. Diggins, V. Schöllmann, T. D. Clark, R. J. Prance, H. Prance, and J. F. Ralph, Phys. Lett. A 234, 205 (1997).

${ }^{18}$ R. Whiteman, V. Schöllmann, M. J. Everitt, T. D. Clark, R. J. Prance, H. Prance, J. Diggins, G. Buckling, and J. F. Ralph, J. Phys.: Condens. Matter 10, 9951 (1998).

${ }^{19}$ J. E. Zimmerman, Proceedings of the 1972 Applied Superconductivity Conference (IEEE Publications, New York, 1972).
${ }^{20}$ R. J. Prance, R. Whiteman, T. D. Clark, H. Prance, V. Schöllmann, J. F. Ralph, S. Al-Khawaja, and M. Everitt, Phys. Rev. Lett. 82, 5401 (1999).

${ }^{21}$ O. V. Lounasmaa, Experimental Principles and Methods Below $1 \mathrm{~K}$ (Academic, London, 1974), pp. 156-159.

${ }^{22}$ E. Ben-Jacob and D. Abraham, Appl. Phys. Lett. 39, 835 (1981).

${ }^{23}$ J. E. Zimmerman, P. Thiene, and J. T. Harding, J. Appl. Phys. 41, 1572 (1970).

${ }^{24}$ S. H. Strogatz, Nonlinear Dynamics and Chaos (Perseus Books, Readings, MA, 1994), p. 211.

${ }^{25}$ L. O. Chua, C. A. Desoer, and E. S. Kuh, Linear and Nonlinear Circuits (McGraw-Hill, New York, 1987), p. 344.

${ }^{26}$ J. M. T. Thompson and H. B. Stewart, Non-linear Dynamics and Chaos (Wiley, Chichester, 1986), p. 84.

${ }^{27} \mathrm{P}$. Glendinning, Stability, Instability, and Chaos (Cambridge University Press, Cambridge, UK, 1994), p. 175.

${ }^{28}$ S. V. Pereverzev, A. Loshak, S. Backhaus, J. C. Davis, and R. E. Packard, Nature (London) 388, 449 (1997).

${ }^{29}$ K. Schwab, N. Bruckner, and R. E. Packard, J. Low Temp. Phys. 110, 1043 (1998).

${ }^{30}$ E. Varoquaux, O. Avenal, G. Ihes, and R. Salmelin, Physica B 178, 309 (1992).

${ }^{31}$ J. F. Ralph, T. D. Clark, J. Diggins, R. J. Prance, H. Prance, and H. Prance, J. Phys.: Condens. Matter 9, 8275 (1997).

${ }^{32}$ See V. P. Koshelets, K. K. Likharev, and V. V. Migulin, IEEE Trans. Magn. 23, 755 (1987); H. Miyake, N. Fukaya, and Y. Okabe, IEEE Trans. Magn. 21, 578 (1985). 